Supporting Information

\title{
Catalytic, Asymmetric Synthesis and Diastereoselective Aldol Reactions of Dipropionate Equivalents
}

Michael A. Calter, Wei Song, and Jianguang Zhou

\begin{tabular}{|c|c|}
\hline Characterization data & $\mathrm{S} 2-\mathrm{S} 4$ \\
\hline $\mathbf{6 a}$ & $\mathrm{S} 2$ \\
\hline $\mathbf{6 b}$ & $\mathrm{S} 2$ \\
\hline 6c & $\mathrm{S} 2$ \\
\hline 6d & $\mathrm{S} 2$ \\
\hline $6 e$ & S3 \\
\hline $6 f$ & S3 \\
\hline 9a & S3 \\
\hline $\mathbf{1 0 a}$ & S3-S4 \\
\hline $10 b$ & $\mathrm{~S} 4$ \\
\hline 10c & $\mathrm{S} 4$ \\
\hline 10d & $\mathrm{S} 4$ \\
\hline
\end{tabular}


[2S, 4R, 5S]- $N$-(5-hydroxy-2,4,6-trimethyl-3-oxo-heptanoyl)-4, $N$-dimethylbenzenesulfonamide (6a). HPLC analysis (Chiralcel OD-H, $0.46 \mathrm{~cm} \times 25 \mathrm{~cm}$, hexane:isopropanol $=96: 4$, flow rate: $0.4 \mathrm{~mL} / \mathrm{min}, \lambda=282 \mathrm{~nm}$ ) of purified 6 a revealed a 96.5:3.5 ratio of enantiomers $\left(\mathrm{R}_{\mathrm{t} 2 S, 4 R, 5 S}=36.2 \mathrm{~min}, \mathrm{R}_{\mathrm{t} 2 R, 4 S, 5 R}=42.1 \mathrm{~min}\right)$. Data for $6 \mathrm{a}:[\alpha]_{\mathrm{D}}^{23}=+11.2^{\circ}(c 0.50$, $\mathrm{CHCl}_{3}$ ); IR(neat film) 3545, 2961, 2876, 1713, 1690, 1596, 1455, 1357, 1164, 982; ${ }^{1} \mathrm{H}$ NMR $\left(\mathrm{CDCl}_{3}, 400 \mathrm{MHz}\right) \delta 7.76(\mathrm{~d}, 2 \mathrm{H}, \mathrm{J}=8.4 \mathrm{~Hz}), 7.38(\mathrm{~d}, 2 \mathrm{H}, \mathrm{J}=8.2 \mathrm{~Hz}), 4.66(\mathrm{q}, 1 \mathrm{H}, \mathrm{J}=7.1 \mathrm{~Hz}), 3.47$ (td, $1 \mathrm{H} \mathrm{J}=8.8,2.6 \mathrm{~Hz}) 3.20$ (s 3H), $2.95(\mathrm{qd}, 1 \mathrm{H}, \mathrm{J}=7.1,2.3 \mathrm{~Hz}), 2.55(\mathrm{~d}, 1 \mathrm{H}, \mathrm{J}=2.9 \mathrm{~Hz}) 2.47$ (s, $3 \mathrm{H}), 1.70-1.65(\mathrm{~m}, 1 \mathrm{H}) 1.37$ (d, 3H, J=7.1 Hz), $1.14(\mathrm{~d}, 3 \mathrm{H}, \mathrm{J}=7.1 \mathrm{~Hz}), 1.04(\mathrm{~d}, 3 \mathrm{H}, \mathrm{J}=6.5 \mathrm{~Hz})$, $1.00(\mathrm{~d}, 3 \mathrm{H}, \mathrm{J}=6.7 \mathrm{~Hz}) ;{ }^{13} \mathrm{C}$ NMR $\left(\mathrm{CDCl}_{3}, 100 \mathrm{MHz}\right) \delta 211.5,171.0,145.4,135.4,130.1,127.5$, 76.1, 53.4, 45.5, 33.2, 30.7, 21.7, 19.4, 18.9, 14.2, 9.3; Anal Calcd for $\mathrm{C}_{18} \mathrm{H}_{27} \mathrm{NO}_{5} \mathrm{~S}: \mathrm{C}, 58.51 ; \mathrm{H}$, 7.37; N, 3.79; Found: C, 58.65; H, 7.36; N, 3.55 .

[2S, $4 R, 5 S]$ - $N$-(5-hydroxy-2,4-dimethyl-3-oxo-octanoyl)-4, $N$-dimethyl-benzenesulfonamide (6b). Isolated as 5.8:1 mixture of diastereomers, data for mixture: IR(neat film) 3498, 2953, 2929, 2857, 1716, 1690, 1461, 1359, 1163, 1090, 993; ${ }^{1} \mathrm{H}$ NMR $\left(\mathrm{CDCl}_{3}, 400 \mathrm{MHz}\right) \delta 7.77$ (d, $2 \mathrm{H}, \mathrm{J}=8.2 \mathrm{~Hz}), 7.38(\mathrm{~d}, 2 \mathrm{H}, \mathrm{J}=8.1 \mathrm{~Hz}), 4.63(\mathrm{q}, 1 \mathrm{H}, \mathrm{J}=7.3 \mathrm{~Hz}), 3.86(\mathrm{~m} \mathrm{1H}), 3.22(\mathrm{~s} 3 \mathrm{H}), 2.74(\mathrm{~m}$, $1 \mathrm{H}), 2.47(\mathrm{~s}, 3 \mathrm{H}), 2.42(\mathrm{~d}, 1 \mathrm{H}, \mathrm{J}=3.1 \mathrm{~Hz}), 1.47-1.44(\mathrm{~m}, 2 \mathrm{H}), 1.34(\mathrm{~d}, 3 \mathrm{H}, \mathrm{J}=7.0 \mathrm{~Hz}), 1.34-1.30$ $(\mathrm{m}, 2 \mathrm{H}), 1.14(\mathrm{~d}, 3 \mathrm{H}, \mathrm{J}=7.1 \mathrm{~Hz}) 0.94(\mathrm{t}, 3 \mathrm{H}, \mathrm{J}=6.8 \mathrm{~Hz}) ;{ }^{13} \mathrm{C} \mathrm{NMR}\left(\mathrm{CDCl}_{3}, 100 \mathrm{MHz}\right) \delta 210.9$, 171.1, 145.4, 135.4, 130.2, 127.5, 71.1, 53.6, 53.3, 48.4, 35.9, 33.3, 21.7, 19.3, 14.0, 10.4; Anal Calcd for $\mathrm{C}_{18} \mathrm{H}_{27} \mathrm{NO}_{5} \mathrm{~S}$ : C, 58.51; H, 7.37; N, 3.79; Found: C,58.53; H,7.45; N, 3.69. [2S, 4R, 5R]- $N$-(5-hydroxy-2,4-dimethyl-3-oxo-5-phenyl-pentanoyl)-4, $N$-dimethylbenzenesulfonamide (6c). $[\alpha]_{\mathrm{D}}{ }^{23}=-1.16^{\circ}\left(c 0.35, \mathrm{CHCl}_{3}\right)$; IR(neat film) $3527,3030,2983,2938$, 1715, 1690, 1596, 1492,1453,1356, 1163, 990; ${ }^{1} \mathrm{H}$ NMR $\left(\mathrm{CDCl}_{3}, 400 \mathrm{MHz}\right) \delta 7.76$ (d, 2H, J=8.4 $\mathrm{Hz}), 7.38-7.28(\mathrm{~m} \mathrm{7H}), 5.03(\mathrm{~m}, 1 \mathrm{H}),, 4.43(\mathrm{q}, 1 \mathrm{H} \mathrm{J}=7.0 \mathrm{~Hz}) 3.19$ (s 3H), $2.97(\mathrm{~m}, 1 \mathrm{H}$, ), 2.76 (d, $1 \mathrm{H}, \mathrm{J}=2.3 \mathrm{~Hz}) 2.45(\mathrm{~s}, 3 \mathrm{H}), 1.22(\mathrm{~d}, 3 \mathrm{H}, \mathrm{J}=7.1 \mathrm{~Hz}), 1.11(\mathrm{~d}, 3 \mathrm{H}, \mathrm{J}=7.0 \mathrm{~Hz}) ;{ }^{13} \mathrm{C} \mathrm{NMR}\left(\mathrm{CDCl}_{3}\right.$, $100 \mathrm{MHz}) \delta 210.3,171.0,145.4,141.5,135.4,130.2,130.1,128.3,127.6,126.0,73.3,53.8$, 51.3, 33.2, 21.7, 13.7, 10.8; Anal Calcd for $\mathrm{C}_{21} \mathrm{H}_{25} \mathrm{NO}_{5} \mathrm{~S}: \mathrm{C}, 62.51 ; \mathrm{H}, 6.24 ; \mathrm{N}, 3.47$; Found: $\mathrm{C}$, $62.32 ; \mathrm{H}, 6.23 ; \mathrm{N}, 3.23$.

[2S, 4R, 5R]- $N$-(6-Benzyloxy-5-hydroxy-2,4-dimethyl-3-oxo-hexanoyl)-4, $N$-dimethylbenzenesulfonamide $(\mathbf{6 d}) \cdot[\alpha]_{\mathrm{D}}^{23}=+4.15^{\circ}\left(c 0.97, \mathrm{CHCl}_{3}\right)$; IR(neat film) $3518,2980,2924$, 2873, 1720, 1690, 1597, 1454, 1358, 1164, 1105, 1090, 994; ${ }^{1} \mathrm{H}$ NMR $\left(\mathrm{CDCl}_{3}, 400 \mathrm{MHz}\right) \delta 7.79$ $(\mathrm{d}, 2 \mathrm{H}, \mathrm{J}=8.3 \mathrm{~Hz}), 7.37-7.31(\mathrm{~m} \mathrm{7H}), 4.64(\mathrm{q}, 1 \mathrm{H}, \mathrm{J}=7.1 \mathrm{~Hz}), 4.53(\mathrm{~d} 2 \mathrm{H}, \mathrm{J}=5.1 \mathrm{~Hz}), 4.03(\mathrm{~m}, 1 \mathrm{H}$ ), $3.50(\mathrm{~m} \mathrm{1H}), 3.41(\mathrm{~m} \mathrm{1H}), 3.20(\mathrm{~s} 3 \mathrm{H}), 2.91(\mathrm{~m}, 1 \mathrm{H}), 2.54(\mathrm{~d}, 1 \mathrm{H}, \mathrm{J}=3.7 \mathrm{~Hz}) 2.45(\mathrm{~s}, 3 \mathrm{H}), 1.32$ $(\mathrm{d}, 3 \mathrm{H}, \mathrm{J}=7.0 \mathrm{~Hz}), 1.14(\mathrm{~d}, 3 \mathrm{H}, \mathrm{J}=7.1 \mathrm{~Hz}) ;{ }^{13} \mathrm{C} \mathrm{NMR}\left(\mathrm{CDCl}_{3}, 100 \mathrm{MHz}\right) \delta 209.1,171.4,145.2$, 137.7, 135.4, 130.0, 129.7, 128.5, 127.8, 127.6, 73.5, 71.3, 71.1, 54.0, 46.7, 33.2, 21.7, 13.8, 12.2; Anal Calcd for $\mathrm{C}_{23} \mathrm{H}_{29} \mathrm{NO}_{6} \mathrm{~S}$ : C, 61.73; H, 6.53; N, 3.13; Found: C, 61.53; H, 6.53; N, 2.93. [2S, 4R, 5S]- $N$-[7-(tert-Butyl-dimethyl-silanyloxy)-5-hydroxy-2,4-dimethyl-3-oxoheptanoyl]-4, $N$-dimethyl-benzenesulfonamide $(6 \mathrm{e}) .[\alpha]_{\mathrm{D}}{ }^{23}=-6.47^{\circ}\left(c 0.85, \mathrm{CHCl}_{3}\right)$; $\mathrm{IR}($ neat film) 3498, 2980, 2953, 2929, 2857, 1716, 1690, 1461, 1359, 1163, 1090, 993; ${ }^{1} \mathrm{H}$ NMR $\left(\mathrm{CDCl}_{3}\right.$, $400 \mathrm{MHz}) \delta 7.82(\mathrm{~d}, 2 \mathrm{H}, \mathrm{J}=8.4 \mathrm{~Hz}), 7.36(\mathrm{~d}, 2 \mathrm{H}, \mathrm{J}=8.2 \mathrm{~Hz}), 4.64(\mathrm{q}, 1 \mathrm{H}, \mathrm{J}=7.1 \mathrm{~Hz}), 4.00(\mathrm{~m}$, $1 \mathrm{H}), 3.86(\mathrm{~m}, 1 \mathrm{H}), 3.78(\mathrm{~m}, 1 \mathrm{H}), 3.64(\mathrm{~d}, 1 \mathrm{H}), 3.24(\mathrm{~s}, 3 \mathrm{H}), 2.8(\mathrm{~m}, 1 \mathrm{H}), 2.46(\mathrm{~s}, 3 \mathrm{H}), 1.56(\mathrm{~m}$, $2 \mathrm{H}), 1.34(\mathrm{~d}, 3 \mathrm{H}, \mathrm{J}=7.1 \mathrm{~Hz}), 1.10(\mathrm{~d}, 3 \mathrm{H}, \mathrm{J}=7.0 \mathrm{~Hz}), 0.9(\mathrm{~s}, 9 \mathrm{H}), 0.08(\mathrm{~d}, 6 \mathrm{H}) ;{ }^{13} \mathrm{C} \mathrm{NMR}\left(\mathrm{CDCl}_{3}\right.$, $100 \mathrm{MHz}) \delta 209.1,171.7,145.2,135.6,130.0,127.7,72.8,62.7,54.6,49.6,35.0,33.2,25.8$, 21.7, 18.1, 13.7, 12.1, -5.5, -5.6; Anal Calcd for $\mathrm{C}_{23} \mathrm{H}_{39} \mathrm{NO}_{6} \mathrm{SSi}$ C, 56.88; H, 8.09; N, 2.88; Found: C, 56.70; H,7.86; N, 2.98 . [2S, 4R, 5R]- $N$-(5-hydroxy-2,4,6-trimethyl-3-oxo-hept-6-enoyl)-4, $N$-dimethylbenzenesulfonamide (6f). $[\alpha]_{\mathrm{D}}{ }^{23}=+15.0^{\circ}\left(c 1.22, \mathrm{CHCl}_{3}\right)$; $\operatorname{IR}($ neat film) $3531,2982,2940,2878$, 
1714, 1691, 1596, 1453, 1356, 1164, 991; ${ }^{1} \mathrm{H} \mathrm{NMR}\left(\mathrm{CDCl}_{3}, 400 \mathrm{MHz}\right) \delta 7.77$ (d, 2H, J=8.4 Hz), $7.38(\mathrm{~d}, 2 \mathrm{H}, \mathrm{J}=8.0 \mathrm{~Hz}), 5.10(\mathrm{~s}, 1 \mathrm{H}), 4.96(\mathrm{~s}, 1 \mathrm{H}), 4.67(\mathrm{q}, 1 \mathrm{H}, \mathrm{J}=7.0 \mathrm{~Hz}), 4.49(\mathrm{~s}, 1 \mathrm{H}), 3.19(\mathrm{~s}$, $3 \mathrm{H}), 2.91(\mathrm{~d}, 1 \mathrm{H}), 2.82(\mathrm{~m}, 1 \mathrm{H}), 2.47(\mathrm{~s}, 3 \mathrm{H}), 1.65(\mathrm{~s}, 3 \mathrm{H}), 1.39(\mathrm{~d}, 3 \mathrm{H}, \mathrm{J}=7.0 \mathrm{~Hz}), 1.01(\mathrm{~d}, 3 \mathrm{H}$, $\mathrm{J}=7.1 \mathrm{~Hz}) ;{ }^{13} \mathrm{C} \mathrm{NMR}\left(\mathrm{CDCl}_{3}, 100 \mathrm{MHz}\right) \delta 210.4,171.0,145.4,143.4,135.4,130.1,127.6,111.9$, 73.5, 53.3, 46.2, 33.2, 21.7, 19.4, 14.1, 9.6; Anal Calcd for $\mathrm{C}_{18} \mathrm{H}_{25} \mathrm{NO}_{5} \mathrm{~S}$ : C, 58.83; H, 6.86; N, 3.81; Found: C,59.10; H,6.81; N, 3.55.

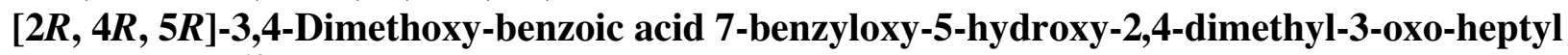
ester (9a). $[\alpha]_{\mathrm{D}}^{23}-17.7^{\circ}\left(c 1.05, \mathrm{CHCl}_{3}\right)$; IR (neat) 3064, 2936, 1714, 1600, 1515, 1456, 1417, 1365, 1346, 1291, 1271, 1222, 1177, 1133, 1108, 1025, 984, $875 \mathrm{~cm}^{-1}$; ${ }^{1} \mathrm{H}$ NMR (400 MHz, $\left.\mathrm{CDCl}_{3}\right) \delta 7.63(\mathrm{dd}, \mathrm{J}=1.9,8.4 \mathrm{~Hz}, 1 \mathrm{H}), 7.51(\mathrm{~d}, \mathrm{~J}=1.9 \mathrm{~Hz}, 1 \mathrm{H}), 7.36-7.30(\mathrm{~m}, 5 \mathrm{H}), 6.89(\mathrm{~d}, \mathrm{~J}=$ $8.4 \mathrm{~Hz}, 1 \mathrm{H}), 4.53$ (s, 3H), $4.48(\mathrm{dd}, \mathrm{J}=8.1,10.9 \mathrm{~Hz}, 1 \mathrm{H}) 4.41(\mathrm{dd}, \mathrm{J}=5.1,10.9 \mathrm{~Hz}, 1 \mathrm{H}), 3.99$ $3.97(\mathrm{~m}, 1 \mathrm{H}), 3.95(\mathrm{~s}, 3 \mathrm{H}), 3.92(\mathrm{~s}, 3 \mathrm{H}), 3.75-3.73(\mathrm{~m}, 1 \mathrm{H}), 3.71-3.66(\mathrm{~m}, 1 \mathrm{H}), 3.21-3.17(\mathrm{~m}$, $1 \mathrm{H}), 2.86$ (apparent quintet, $\mathrm{J}=7.2 \mathrm{~Hz}, 1 \mathrm{H}), 1.86-1.82(\mathrm{~m}, 1 \mathrm{H}), 1.78-1.73(\mathrm{~m}, 1 \mathrm{H}), 1.18(\mathrm{~d}, \mathrm{~J}=$ $7.0 \mathrm{~Hz}, 3 \mathrm{H}), 1.10(\mathrm{~d}, \mathrm{~J}=7.1 \mathrm{~Hz}, 3 \mathrm{H}) ;{ }^{13} \mathrm{C} \mathrm{NMR}\left(100 \mathrm{MHz}, \mathrm{CDCl}_{3}\right) \delta$ 215.8, 166.0, 153.0, 148.6, 137.8, 128.4, 127.8, 127.7, 123.5, 122.3, 111.8, 110.2, 73.4, 72.8, 68.6, 65.6, 56.0, 55.9, 50.8, 45.5, 33.9, 13.2, 13.1. Anal. Calcd for $\mathrm{C}_{25} \mathrm{H}_{32} \mathrm{O}_{7}: \mathrm{C}, 67.55 ; \mathrm{H}, 7.26$. Found: C, 67.82; H, 7.30. [2R,4R,5R]-3,4-Dimethoxy-benzoic acid 5-hydroxy-2,4,6-trimethyl-3-oxo-heptyl ester (10a). $[\alpha]_{\mathrm{D}}{ }^{23}=-11.5^{\circ}\left(c 1.34, \mathrm{CHCl}_{3}\right)$; IR(neat film) 3535, 2964, 2878, 1711, 1599, 1515, 1462, 1417, 1371, 1346, 1271, 1223, 1177, $1023 \mathrm{~cm}^{-1}$; ${ }^{1} \mathrm{H}$ NMR $\left(\mathrm{CDCl}_{3}, 400 \mathrm{MHz}\right) \delta 7.62$ (dd, 1H, J=1.9, 8.4 Hz), 7.49 (d, 1H, J=1.9 Hz), 6.87 (d, 1H, J=8.5 Hz), 4.47 (dd, 1H, J=8.3,10.9 Hz), 4.35 (dd, 1H, $\mathrm{J}=5.1,11.3 \mathrm{~Hz}), 3.93(\mathrm{~s}, 3 \mathrm{H}) 3.91(\mathrm{~s}, 3 \mathrm{H}), 3.53(\mathrm{~m}, 1 \mathrm{H}), 3.22-3.17(\mathrm{~m}, 1 \mathrm{H}) 2.96-2.89(\mathrm{~m}, 1 \mathrm{H})$, $2.38(\mathrm{~d}, 1 \mathrm{H} \mathrm{J}=6.3 \mathrm{~Hz}), 1.80-1.72(\mathrm{~m}, 1 \mathrm{H}), 1.18(\mathrm{~d}, 3 \mathrm{H}, \mathrm{J}=7.1 \mathrm{~Hz}), 1.11(\mathrm{~d}, 3 \mathrm{H}, \mathrm{J}=7.2 \mathrm{~Hz}), 0.97$ $(\mathrm{d}, 3 \mathrm{H}, \mathrm{J}=6.8 \mathrm{~Hz}), 0.91(\mathrm{~d}, 3 \mathrm{H}, \mathrm{J}=6.8 \mathrm{~Hz}) ;{ }^{13} \mathrm{C} \mathrm{NMR}\left(\mathrm{CDCl}_{3}, 100 \mathrm{MHz}\right) \delta 216.6,166.0,153.1$, 148.6, 123.5, 122.3, 111.9, 110.2, 77.8, 65.5, 56.0, 55.9, 48.0, 45.3, 30.1, 20.0, 15.5, 13.7, 13.4; Anal Calcd for $\mathrm{C}_{19} \mathrm{H}_{28} \mathrm{O}_{6}: \mathrm{C}, 64.75 ; \mathrm{H}, 8.01$; Found: C, 64.69; H, 8.29.

[2R,4R,5R]-3,4-Dimethoxy-benzoic acid 5-hydroxy-2,4-dimethyl-3-oxo-octyl ester (10b). White solid, m.p. $78-79^{\circ} \mathrm{C} ;[\alpha]_{\mathrm{D}}{ }^{23}=-5.00^{\circ}\left(c 1.80, \mathrm{CHCl}_{3}\right)$; IR(neat film) 3408, 3343, 2953, 2894, 2872, 1708, 1596, 1515, 1463, 1414, 1375, 1345, 1275, 1222, 1180, $1017 \mathrm{~cm}^{-1} ;{ }^{1} \mathrm{H}$ NMR $\left(\mathrm{CDCl}_{3}\right.$, $400 \mathrm{MHz}) \delta 7.60(\mathrm{dd}, 1 \mathrm{H}, \mathrm{J}=1.8,8.0 \mathrm{~Hz}), 7.47(\mathrm{~d} 1 \mathrm{H}, \mathrm{J}=1.8 \mathrm{~Hz}), 6.86(\mathrm{~d}, 1 \mathrm{H}, \mathrm{J}=8.4 \mathrm{~Hz}), 4.46$ $(\mathrm{dd}, 1 \mathrm{H}, \mathrm{J}=8.5,10.8 \mathrm{~Hz}), 4.33(\mathrm{dd}, 1 \mathrm{H}, \mathrm{J}=5.0,10.9 \mathrm{~Hz}), 3.92(\mathrm{~s}, 3 \mathrm{H}), 3.89(\mathrm{~s}, 3 \mathrm{H}), 3.72(\mathrm{~m}, 1 \mathrm{H})$, 3.21-3.12 (m, 1H), 2.81-2.74 (m, 1H), $2.60(\mathrm{~s}, 1 \mathrm{H}), 1.55-1.44(\mathrm{~m}, 4 \mathrm{H}), 1.16(\mathrm{~d}, 3 \mathrm{H}, \mathrm{J}=7.1 \mathrm{~Hz})$, $1.13(\mathrm{~d}, 3 \mathrm{H}, \mathrm{J}=7.2 \mathrm{~Hz}), 0.91(\mathrm{t}, 3 \mathrm{H}, \mathrm{J}=7.0 \mathrm{~Hz}) ;{ }^{13} \mathrm{C} \mathrm{NMR}\left(\mathrm{CDCl}_{3}, 100 \mathrm{MHz}\right) \delta 216.6,166.0$, 153.0, 148.6, 123.5, 122.3, 111.8, 110.2, 73.1, 65.3, 56.0, 55.9, 50.7, 45.1, 36.7, 18.7, 13.9, 13.7, 13.3; Anal Calcd for $\mathrm{C}_{19} \mathrm{H}_{28} \mathrm{O}_{6}$ : C, 64.75; H, 8.01; Found: C, 64.54; H, 8.03.

[2R,4R,5S]-3,4-Dimethoxy-benzoic acid 5-hydroxy-2,4-dimethyl-3-oxo-5-phenyl-pentyl ester (10c). White solid, m.p. $39-40^{\circ} \mathrm{C} ;[\alpha]_{\mathrm{D}}{ }^{23}=-39.2^{\circ}\left(c 2.00, \mathrm{CHCl}_{3}\right)$; IR(neat film) 3513,2971 , 2936, 1711, 1598, 1514, 1458, 1417, 1348, 1271, 1223, 1177, $1024 \mathrm{~cm}^{-1} ;{ }^{1} \mathrm{H}$ NMR $\left(\mathrm{CDCl}_{3}, 400\right.$ $\mathrm{MHz}) \delta 7.60(\mathrm{dd}, 1 \mathrm{H}, \mathrm{J}=1.9,8.4 \mathrm{~Hz}), 7.47(\mathrm{~d}, 1 \mathrm{H}, \mathrm{J}=1.9 \mathrm{~Hz}), 7.37-7.27(\mathrm{~m}, 5 \mathrm{H}), 6.86(\mathrm{~d}, 1 \mathrm{H}$, $\mathrm{J}=8.4 \mathrm{~Hz}), 4.79$ (dd, 1H, J=3.8, 8.0 Hz), 4.47 (dd, 1H, J=8.1, 10.9 Hz), 4.35 (dd, 1H, J=5.1, 10.9 $\mathrm{Hz}), 3.92(\mathrm{~s}, 3 \mathrm{H}), 3.88(\mathrm{~s}, 3 \mathrm{H}), 3.22-3.16(\mathrm{~m}, 1 \mathrm{H}), 3.16-3.06(\mathrm{~m}, 1 \mathrm{H}), 2.88(\mathrm{~d}, 1 \mathrm{H} \mathrm{J}=4.3 \mathrm{~Hz})$, $1.11(\mathrm{~d}, 3 \mathrm{H}, \mathrm{J}=7.1 \mathrm{~Hz}), 0.95(\mathrm{~d}, 3 \mathrm{H}, \mathrm{J}=7.2 \mathrm{~Hz}) ;{ }^{13} \mathrm{C} \mathrm{NMR}\left(\mathrm{CDCl}_{3}, 100 \mathrm{MHz}\right) \delta 215.9,166.0$, 153.0, 148.6, 142.1, 128.5, 128.0, 126.5, 123.5, 122.3, 111.8, 110.2, 76.4, 65.4, 56.0, 55.9, 52.4, 45.7, 14.1, 12.9; Anal Calcd for $\mathrm{C}_{22} \mathrm{H}_{26} \mathrm{O}_{6}$ : C, 68.38; H, 6.78; Found: C, 67.09; H, 6.29.

[2R,4R,5R]-3,4-Dimethoxy-benzoic acid 5-hydroxy-2,4,6-trimethyl-3-oxo-hept-6-enyl ester (10d). White solid, m.p. $68-70^{\circ} \mathrm{C} ;[\alpha]_{\mathrm{D}}^{23}=-28.1^{\circ}\left(c 1.62, \mathrm{CHCl}_{3}\right)$; $\mathrm{IR}(\mathrm{KBr}) 3517,2972,2936$, $1711,1599,1514,1459,1417,1371,1348,1271,1223,1177,1024 \mathrm{~cm}^{-1} ;{ }^{1} \mathrm{H} \mathrm{NMR}\left(\mathrm{CDCl}_{3}, 400\right.$ 
MHz) $\delta 7.60$ (dd, 1H, J=1.8, 8.6 Hz), 7.48 (d, 1H, J=1.4 Hz), 6.86 (d, 1H, J=8.4 Hz), 4.94 (s 1H), $4.91(\mathrm{~s}, 1 \mathrm{H}), 4.47(\mathrm{dd}, 1 \mathrm{H}, \mathrm{J}=8.0,10.8 \mathrm{~Hz}), 4.35(\mathrm{dd}, 1 \mathrm{H}, \mathrm{J}=5.1,10.9 \mathrm{~Hz}), 4.22(\mathrm{~d}, 1 \mathrm{H}, \mathrm{J}=8.2$

$\mathrm{Hz}), 3.92(\mathrm{~s}, 3 \mathrm{H}), 3.90(\mathrm{~s}, 3 \mathrm{H}), 3.23-3.15(\mathrm{~m}, 1 \mathrm{H}), 2.98-2.90(\mathrm{~m}, 1 \mathrm{H}), 2.39(\mathrm{~s}, 1 \mathrm{H}), 1.73(\mathrm{~s}, 3 \mathrm{H})$, $1.23(\mathrm{~d}, 3 \mathrm{H}, \mathrm{J}=8.4 \mathrm{~Hz}), 0.98(\mathrm{~d}, 3 \mathrm{H}, \mathrm{J}=7.1 \mathrm{~Hz}) ;{ }^{13} \mathrm{C} \mathrm{NMR}\left(\mathrm{CDCl}_{3}, 100 \mathrm{MHz}\right) \delta$ 215.5, 166.0, 153.0, 148.5, 144.5, 123.5, 122.3, 114.2, 111.8, 110.2, 78.2, 65.5, 55.9, 55.9, 48.1, 45.7, 16.7, 13.8, 13.0; Anal Calcd for $\mathrm{C}_{19} \mathrm{H}_{26} \mathrm{O}_{6}$ : C, 65.13; H, 7.48; Found: C, 65.09; H, 7.53. 